\title{
Machining Parameters and Toolpath Productivity Optimization Using a Factorial Design and Fit Regression Model in Face Milling and Drilling Operations
}

\author{
Gustavo M. Minquiz $\mathbb{D},{ }^{1,2}$ Vicente Borja $\mathbb{D}^{3},{ }^{3}$ Marcelo López-Parra $\mathbb{D}^{3},{ }^{3}$ \\ Alejandro C. Ramírez-Reivich $\mathbb{D D}^{3}{ }^{3}$ Leopoldo Ruiz-Huerta, ${ }^{4,5}$ R. C. Ambrosio Lázaro $\mathbb{D}^{1}{ }^{1}$ \\ Alejandro Shigeru Yamamoto Sánchez $\mathbb{D}^{6},{ }^{6}$ H. Vazquez-Leal $\mathbb{D}^{7}$, \\ María-Esther Pavon-Solana $\mathbb{D},{ }^{1}$ and J. Flores Méndez $\mathbb{D}^{1,2}$ \\ ${ }^{1}$ Benemérita Universidad Autónoma de Puebla-Ciudad Universitaria, Blvd. Valsequillo y Esquina, Av. San Claudio $s / n$, \\ Col. San Manuel, C.P. 72570, Puebla, Pue, Mexico \\ ${ }^{2}$ Tecnológico Nacional de México/I.T, Puebla Av. Tecnológico No. 420, Maravillas, C.P 72220, Puebla, Pue, Mexico \\ ${ }^{3}$ Universidad Nacional Autónoma de México, Facultad de Ingeniería, Av. Universidad No. 3000, C.P. 04510, \\ Ciudad de México, Mexico \\ ${ }^{4}$ Universidad Nacional Autónoma de México, Instituto de Ciencias Aplicadas y Tecnología (ICAT), Circuito Exterior s/n, \\ Ciudad Universitaria AP 70-186, C.P. 04510, Ciudad de México, Mexico \\ ${ }^{5}$ National Laboratory for Additive and Digital Manufacturing, (MADiT), Circuito Exterior $s / n$, Ciudad Universitaria, \\ A.P. 70-186, C.P 04510, Ciudad de México, Mexico \\ ${ }^{6}$ Sandvik Coromant México-Parque Industrial Querétaro, Av. Cerrada de la Estacada \#550 C, Santa Rosa Jaúregui, \\ C.P. 76220, Mexico \\ ${ }^{7}$ Facultad de Instrumentación Electrónica, Universidad Veracruzana, Cto. Gonzalo Aguirre Beltrán S/N 91000, \\ Xalapa-Veracruz, Mexico
}

Correspondence should be addressed to J. Flores Méndez; xavier_snk@hotmail.com

Received 10 February 2020; Revised 29 May 2020; Accepted 30 May 2020; Published 27 July 2020

Guest Editor: Mohamed Nayel

Copyright $(2020$ Gustavo M. Minquiz et al. This is an open access article distributed under the Creative Commons Attribution License, which permits unrestricted use, distribution, and reproduction in any medium, provided the original work is properly cited.

\footnotetext{
Very commonly, a mechanical workpiece manufactured industrially includes more than one machining operation. Even more, it is a common activity of programmers, who make a decision in this regard every time a milling and drilling operation is performed. This research is focused on better understanding the power behavior for face milling and drilling manufacturing operations, and the methodology followed was the design of experiments (DOEs) with the cutting parameters set in combination with toolpath evaluation available in commercial software, having as main goal to get a predictive power equation validated in two ways, linear or nonlinear, and understanding the energy consumption and the quality surface in face milling and final diameter in drilling. The results show that it is possible to find difference in a power demand of $1.52 \mathrm{~kW}$ to $3.9 \mathrm{~kW}$ in the same workpiece, depending on the operations (face milling or drilling), cutting parameters, and toolpath chosen. Additionally, the equations modelled showed acceptable values to predict the power, with $p$ values higher than 0.05 which is the significance level for the nonlinear and linear equations with an $R$ square predictive of 98.36 . Some conclusions established that optimization of the cutting parameters combined with toolpath strategies can represent an energy consumption optimization higher than $0.21 \%$ and the importance to try to find an energy consumption balance when a workpiece has different milling operations.
} 


\section{Introduction}

According to Energy Information Administration (EIA) [1], 27203 Trillion BTU was the total energy consumed by the industrial sector in 2018. The aerospace, automotive, and plastics industries use machining processes to produce metallic parts for different manufacturing purposes [2]. To improve energy consumption efficiency in manufacturing systems, several researchers have focused their work on identifying the primary variables involved in machining [3]. Several analyses to assess the environmental impacts of machining processes have been carried out to better understand the behavior of the energy consumed. Some authors have found that the energy required for material removal is very low when compared to the total energy of the machine tool operation [4]. Therefore, several researchers have focused their work on finding ways to reduce this total energy rate. Guo et al. [5-7] analyzed the surface quality and energy consumption of a turning process; the aforementioned research tested a workpiece made of steel 11SMnPb30 and found that the best cutting parameters ranged as follows: a table feed of $0.05-0.3 \mathrm{~mm} / r$, an axial depth of cut $0.5-1 \mathrm{~mm}$, and a cutting speed of $80-800 \mathrm{~m} / \mathrm{min}$. To understand and minimize energy behavior in the milling processes, different operations have been studied. These previous works have proposed face milling improvements based on efficient toolpaths and energy consumption [8-10]. These improvements were such as the optimization of surface milling regarding cycle time, as well as the study of toolpath selection as a critical factor $[11,12]$. Investigation in the optimization of machining parameters trying A6061 aluminum and using the response surface methodology based on particle swarm optimization not only led to finding an optimal combination of machining parameters but also that the spindle speed variable is the most influencing in surface roughness, power consumption, and cutting force. This alternative study showed $\pm 7 \%$ of error [13]. Mathematical power prediction has been proposed, based on experimental models of infinitesimal cutting force during the machining process in order to obtain the power consumption, where the data were divided into spindle rotational, feed motion, and idle power, and the error of mean power was $0.208 \%$ [14]. Other studies aimed at the development of a mathematical model to know the power demand in face milling has considered both the cutting parameters and the flank wear [15]. Additionally, an artificial intelligence model was proposed in order to monitor the main drive power in the CNC machine in real time. The surface roughness and measuring the flank wear of the cutting tool were the main output variables considered, and random forest techniques had an accuracy between 33 and $44 \%$ [16]. Other techniques have considered the multioptimization analysis, using the same roughness surface methodology, using titanium Ti-6A1-4V, as well as the surface roughness values. This model made it possible to know the power consumption with a correlation coefficient higher than 90\% [17]. Other relevant studies consider the laser surface texturing (LST) technique in combination with the addition of nanoparticles (NPs) to the cutting fluid for getting better results in terms of reducing the spindle load (power demand) and reducing the tool wear in relation to surface roughness and thus finding that it is possible to improve the milling process up to $11 \%$ [18]. Some studies have successfully used milling processes with more than one machining type, determining different cutting parameters for the primary geometry features of their case studies $[19,20]$. Behrendt et al. [19] propose a procedure for machine tool selection based on energy consumption, while Helu et al. [20] evaluate green machining technologies based on sustainability and cost. Li et al. [21] have investigated workpieces with different machined features to identify relevant factors. They found that critical factors are cutting parameters optimization, process planning, and job shop scheduling. The analysis that they have carried out has proved that the toolpath selection impacts both the material removal power and the air cutting times. For a process, which includes more than one milling feature, it is necessary to select different cutting parameters for each feature, based on material, cutting tool, and toolpath $[22,23]$. The power required by a milling operation depends on parameters like spindle speed, feed rate, depth of cut, and transversal cut [24]. Different investigations have proposed energy consumption predictive models based on cutting parameters to estimate surface roughness [25, 26] and tool wear [27]. In order to optimize machining processes, different statistical techniques were used. With the use of either nonlinear or linear polynomial models, it is possible to model power behavior. Amongst these techniques are design of experiments (DOEs), Taguchi, nondominated sorting genetic algorithm (NSGA), and response surface.

This paper reports on a research aimed at modelling the power consumption of the face milling and drilling operations in terms of cutting parameters. This is relevant because such power demand models assist in defining cutting parameters in order to achieve the required quality of surface, which is key in manufacturing processes and adequate productivity rate. The energy consumption is also considered as a factor. Besides, the work reported successfully identifies facing and drilling toolpaths with the lowest power demands and employs DOE to define tests that were carried out to calculate the constants of the desired models. The methodology used by the authors identified that nonlinear equations provided a better fit than linear equations for the machining operations studied. Additionally, the combination of the cutting parameters and toolpath is more suitable to improve a machining process.

\section{Power Demand Background}

Several researchers have worked on understanding power consumption on machine tools. Draganescu et al. attempted to develop a model for the spindle motor's efficiency $\eta$ using the following equation, which relates the $p_{c}$, minimal cutting power, with $p_{m c}$, the power consumed by the spindle drive motor [28]:

$$
\eta=\frac{p_{c}}{p_{\mathrm{mc}}}
$$


Gutowski et al. [4] determined the power consumed in a machining operation using the following equation, which estimates $p$, total power, with $p_{o}$, idle power, $\dot{m}$, the rate of material processing, and $k$, a constant:

$$
p=p_{o}+k \dot{m} \text {. }
$$

Different authors $[4,7]$ have analyzed machining power demand composed of three components:

(i) Standby Power. It is the constant power consumed by a machine tool whether material is being cut or not. The electrical components of a machine that always consume power are its controller, its computer panel, and its lights.

(ii) Component Power. It is the power required to achieve the desire spindle speed and feed rate and is needed to carry out each tool change and power the coolant pump.

(iii) Machining Power. It is the power used to remove material.

There exist different alternatives to name each part of the power used by a CNC machine. For example, equation (3) shows the total power consumed by a machine tool. The constant power is the equivalent to the standby state which includes unloaded motor, coolant pump, computer, and fans. The variable power includes the required power to move the spindle and carrying out jog and tool change. Finally, the force necessary to remove the material is the cutting power, Cutting $P$ in equation (3), which is equivalent to the machining power. On the other hand, the constant power, Constant $P$, plus the variable power, Variable $P$, equal the tare power [4]:

$$
T \text {. Power }=\text { Constant } P+\text { Variable } P+\text { Cutting } P \text {. }
$$

2.1. Models to Predict Surface Roughness and Power Consumption. Different statistical techniques $[29,30]$ have been used to model the relationship between output variables and cutting parameters in machining processes. These techniques have showed their advantages on accuracy and simplicity. To establish a regression model for a particular phenomenon or behavior, it is necessary to set up selective experiments, considering output variables as dependent and input variables as independent [31]. Next, data are collected from running experiments, and an equation, i.e., a regression model, is defined based on a statistical analysis of measurements. There exist several regression models, i.e., first order, second order, and nonlinear models. In the particular case of nonlinear models, their constants can be calculated using the method of least square on the results of experiments [31]. Fang and Safi-Jahanshahi [26] proposed a nonlinear model (fit regression model), to predict the surface roughness as a variable dependent on the cutting speed, feed per tooth, and depth of cut, as follows:

$$
y=x_{1} \cdot a^{x_{2}} \cdot b^{x_{3}} \cdot c^{x_{4}} .
$$

In equation (4), the dependent variable is $y ; x_{1}, x_{2}, x_{3}$, and $x_{4}$ are constants; and $a, b$, and $c$ are independent variables. The experiments documented in [26] reported an average error for equation (4) of $3.86 \%$, and constants $x_{1}, x_{2}, x_{3}$, and $x_{4}$ started with a value of one. The Levenberg-Marquardt algorithm was used to find the optimum fit values. The following equation was used to solve the Levenberg-Marquardt algorithm:

$$
\delta(k)=\left(V^{T} V+k D\right)^{-1} V^{T}(y-\eta),
$$

where $k$ is a conditioning factor and $D$ is a diagonal matrix with elements of $V^{T} V$. The direction of $\delta(k)$ is intermediate between the direction of the Gauss-Newton increment $(k \longrightarrow 0)$ and the direction of steepest descent, as follows:

$$
\frac{V^{T}(y-\eta)}{\left\|V^{T}(y-\eta)\right\|}, \quad(k \longrightarrow \infty)
$$

This paper contributes to the understanding of the power and energy behaviors as functions of machining process strategies and cutting parameters. Two milling operations are studied, facing and drilling. In order to identify which one consumes less power, three strategies are tested for facing and two for drilling. Next, DOE is used to define experiments, and the Levenberg-Marquardt algorithm with a least square estimation is employed to help in calculating the constants of linear and nonlinear models that lead to the final total power estimation. Additionally, measurements of surface finish achieved during the experiments and a nonlinear model for tare power are reported.

\section{Experiment Setup}

Figure 1 shows the use of the DOE methodology followed in this work to model the energy consumed by face milling and drilling.

\subsection{Case Study Specification and Cutting Tool Selection.}

The process comprised two operations: first, face milling, consistent on getting a flat area, perpendicular to the axis of the spindle, with a depth of cut of $3 \mathrm{~mm}$; second, a drilling operation applied on nine $\varnothing 14 \mathrm{~mm}$ holes with a depth of $25 \mathrm{~mm}$, in a rectangular array of $3 \times 3$. The machining process to manufacture the part was defined using the commercial software Mastercam ${ }^{\circledR} \times 8$. The face milling operation was performed using different toolpath strategies for each of them: dynamic, zigzag, and one-way. This operation was carried out in one step, i.e., only one facing stage, while the drilling operation was defined first using the counterbore toolpath cutting option and then employing the chip break. Table 1 shows the experiment about material and cutting tool.

The experiments here reported (Tables 2 and 3) implemented the use of air to remove chips during the process because the color of the chips was blue, which meant that due to the settings selected for the cutting parameters, the friction between the tool and the material was not too high. 


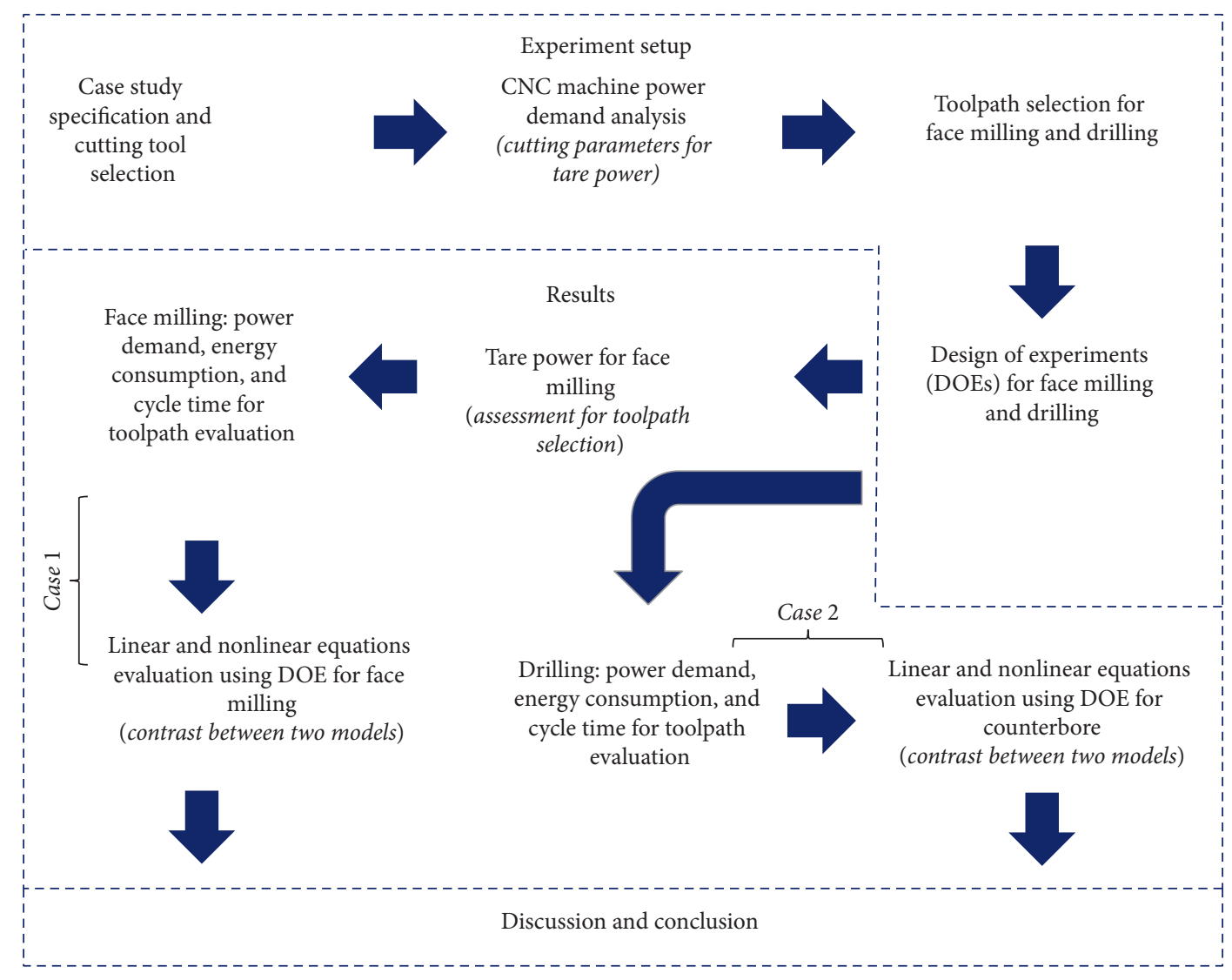

FIGURE 1: Methodology followed for this research.

TABLE 1: Details of the experiment.

Workpiece

dimensions

Workpiece material

Chemical composition

Tool material
$180 \mathrm{~mm} \times 150 \mathrm{~mm}$, depth $30 \mathrm{~mm}$, AISI 1045 medium carbon steel, hardness, Brinell 163

Carbon $0.43-0.5 \%$ max, manganese $0.6-0.9 \%$ max, silicon $0.05 \% \max$, phosphorus $0.04 \%$ max, and sulphur $0.050 \%$ $\max$

ISO code IP240-1200-XA 1630 a solid carbide square end mill with effective cutting edge: 4, cutting diameter: $12 \mathrm{~mm}$, flute helix angle: $35 \mathrm{deg}$, corner chamfer width: $0.2 \mathrm{~mm}$

ISO code 860.1-1400-040A1-PM 4234 a solid carbide drill with cutting diameter: $14 \mathrm{~mm}$, hole tolerance: H8, point angle: $147^{\circ}$, chip flute length: $60 \mathrm{~mm}$
TABLE 2: Cutting parameters used in the DOE for tare power.

\begin{tabular}{lccc}
\hline Parameters & 1 & 2 & 3 \\
\hline Spindle speed $(\mathrm{rpm})$ & 2005 & 5777 & 9549 \\
Feed rate $(\mathrm{mm} / \mathrm{min})$ & 802 & 2607 & 4412 \\
\hline
\end{tabular}

\subsection{CNC Machine Power Demand Analysis, Surface Rough-} ness, and Drilling Holes. The experiments were set up on a milling machine Haas VM3. The power was measured using a three-phase energy analyzer Fluke ${ }^{\circledR} 430$ series II, which is a portable device, complemented by alligator clips and current clamps to connect it (Figure 2). The measurement equipment took data every second, sending information to the computer, and analyzed in the Fluke ${ }^{\circledR}$ PowerLog software.

A portable surface roughness tester Mitutoyo ${ }^{\circledR}$ SJ-210 (Figure 2) was used; the instrument was set to measure under ISO $4287: 1997$ the arithmetical mean roughness value ( $\mathrm{Ra})$
TABLE 3: Cutting parameters for the DOE for the face milling and drilling operations.

\begin{tabular}{lcccc}
\hline & Parameters & \multicolumn{3}{c}{ Level } \\
& & Low & Medium & High \\
\hline \multirow{4}{*}{ Face milling } & $v_{c}$, cutting speed $(\mathrm{m} / \mathrm{min})$ & 315 & 350 & 385 \\
& $a_{p}$, depth of cut $(\mathrm{mm})$ & 0.5 & 1.0 & 2.0 \\
& $f_{z}$, feed per tooth $(\mathrm{mm})$ & 0.15 & 0.175 & 0.2 \\
\hline \multirow{2}{*}{ Drilling } & Cutting speed $(\mathrm{m} / \mathrm{min})$ & 315 & 350 & 385 \\
& Feed $(\mathrm{mm} / r)$ & 0.15 & 0.175 & 0.2 \\
\hline
\end{tabular}

with a resolution of $0.006 \mu \mathrm{m}$, cutoff wavelength $\lambda c$ of 0.8 , roughness sampling length of $0.8 \mathrm{~mm}$, the mean groove spacing RSm (mean peak width) of $\times 5$ and $0.05 \mathrm{~mm} / \mathrm{s}$ speed, stylus tip radius of $5 \mu \mathrm{m}$, and measuring force of $4 \mathrm{mN}$ to measure the surface quality. The procedure can be described as follows: the first step consists on taking three samples in 


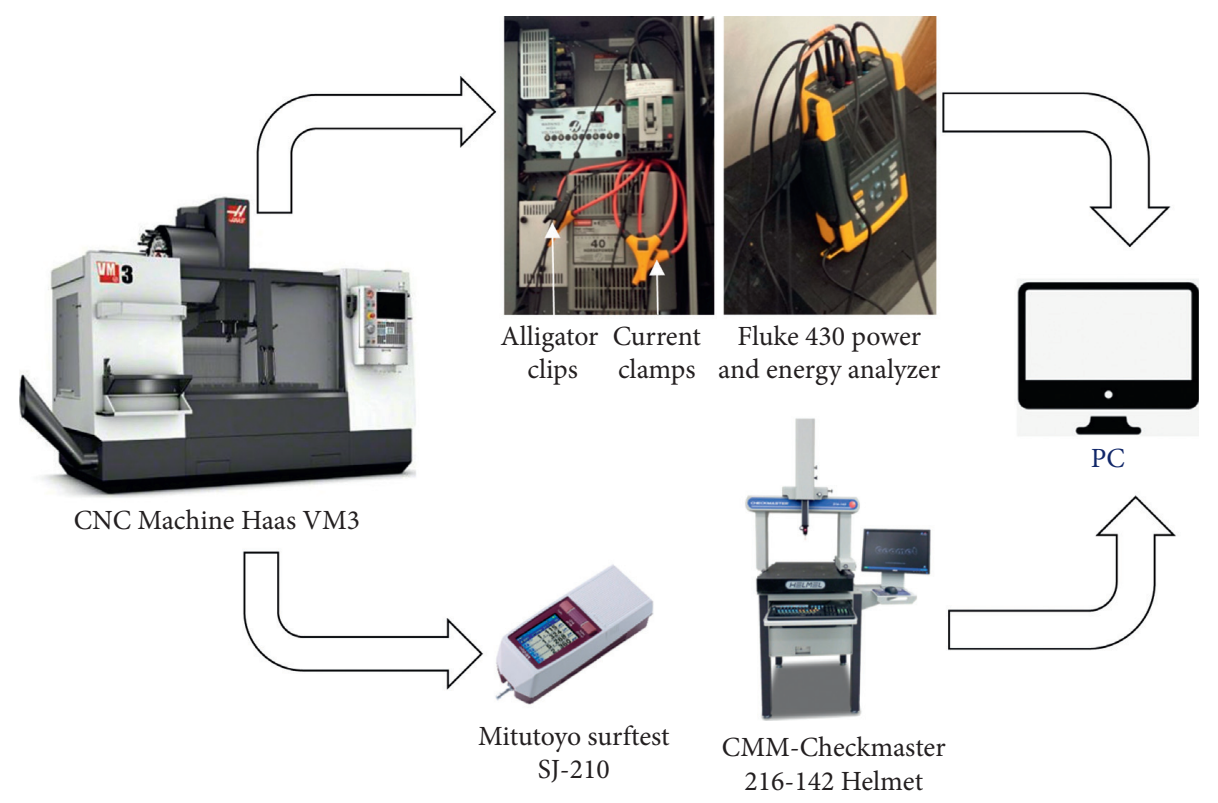

Figure 2: Schematic representation for collecting data.

different points along the flat facing machined area; the second step is to repeat three times the measure in the same points and finally is getting the average between the measurements that were obtained. The drilling holes were measured with a Checkmaster ${ }^{\circledR} 216$ 142-Helmet with resolution (Figure 2) $0.5 \mu \mathrm{m}$, coordinate measuring machine using a Renishaw sensor adapted to M2 $\varnothing 4 \mathrm{~mm}$ ruby ball, stainless steel stem, and $L 20 \mathrm{~mm}$; the data were collected and analyzed using Geomet ${ }^{\circledR} 101$ software. The procedure followed begins with collecting the first 3 data points at or near the bottom along the axis of a cylinder, secondly collecting the last 3 data points at or near the top, then repeating twice the measure in the same points, and finally getting the average between the measurements found.

The breakdown of the power of the CNC machine used was analyzed using the measurements that were obtained with the power analyzer.

3.2.1. Total Power $(k W)$. The total power consumed in each one of the experiments (Tables 2 and 3) was measured. Different tests in each experiment were carried out to estimate the coefficients $x_{1}, x_{2}$, and $x_{3}$, while $a_{p}, v_{c}$, and $f_{z}$ are values of the cutting parameters chosen by the user to model the power consumption with a first-order model (see equation (7)) and a nonlinear model (fit regression model) (see equation (8)) and identifying the convenience of both:

$$
\begin{aligned}
& \mathrm{kW}_{\text {Total }}=a_{p} \cdot x_{1}+v_{c} \cdot x_{2}+f_{z} \cdot x_{3}, \\
& \mathrm{~kW}_{\text {Total }}=x_{1} \cdot a_{p}^{x_{2}} \cdot v_{c}^{x_{3}} \cdot f_{z}^{x_{4}} .
\end{aligned}
$$

3.2.2. Constant Power. His factor was measured setting the machine to the standby state. Its value was $0.55 \mathrm{~kW}$. During the experiments, the coolant water pump was turned off because the metal chips were removed by using air instead.

3.2.3. Tare Power. Face milling was used for modelling the tare power. It was measured setting the machine tool in a running condition, without removing material configured with the dynamic toolpath strategy. This toolpath was used in the study (Table 2) considering the low, middle, and high ranges that could reach the spindle speed and feed rate. So, nine tests were defined in this experiment. The data collected were used to calculate the constants of the nonlinear model proposed to estimate this energy. The model was established with spindle speed, $n$, and feed rate, $v_{f}$, as independent variables, shown as follows:

$$
\mathrm{kW}_{\text {Tare }}=x_{1}+n^{x_{2}}+v_{f}^{x_{3}} \text {. }
$$

3.2.4. Cutting Power. This is the necessary power to remove material. It could be determined by subtracting the tare power from the total power.

The parameters chosen in Table 2 were set under parameters mentioned in the data sheet of the CNC machine, the maximum spindle speed is $1200 \mathrm{rpm}$, while the maximum feed rate is $18000 \mathrm{~mm} / \mathrm{min}$, and also the parameters are according to cutting tool operations suggested by the supplier. The coolant was turned off because it was not removed material.

3.3. Toolpath Selection for Face Milling and Drilling. This research considered the face milling for modelling the tare power. Cutting parameters were set as recommended by the tool supplier [32] for the facing operation. Three toolpath strategies were used: dynamic, zigzag, and one-way (Figure 3); the toolpath with the lowest power demand was identified. 


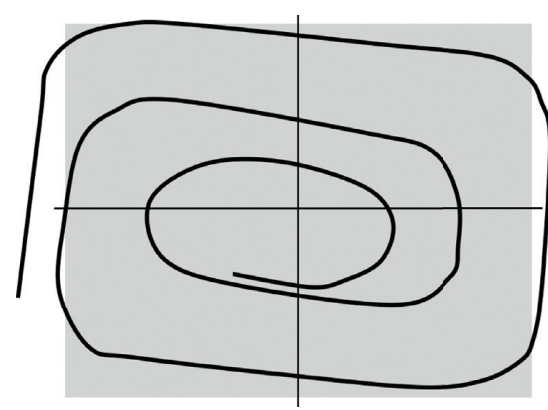

(a)

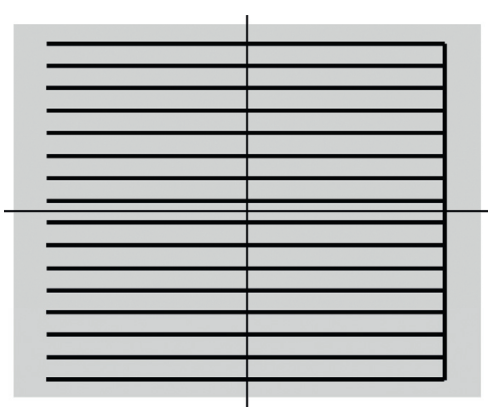

(b)

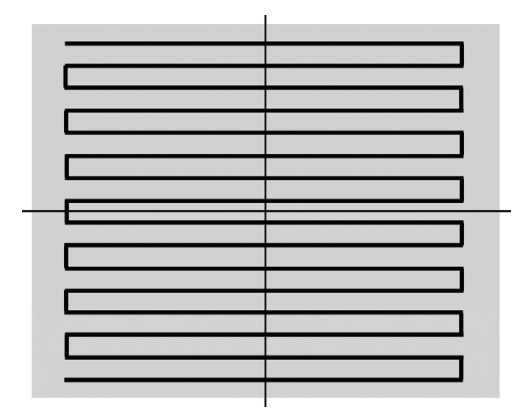

(c)

Figure 3: Toolpath strategies: (a) dynamic; (b) one-way; (c) zigzag. The drill counterbore and chip break toolpath cutting options were tested to identify the tool path strategy that required the lowest power demand.

The cutting parameters were the same for the three strategies (i.e., feed per tooth $0.2 \mathrm{~mm}$, cutting speed $385 \mathrm{~m} / \mathrm{min}$, and depth of cut $0.5 \mathrm{~mm}$ ), reaching a final depth of $3.0 \mathrm{~mm}$.

\subsection{Design of Experiments for Face Milling and Drilling.} The cutting parameters for face milling were defined using a DOE $3^{3}$. Table 3 shows these values. In order to obtain a satisfactory roughness, the levels of the depth of cut were set to low values: $0.5,1$, and, finally, $2 \mathrm{~mm}$. The quality of surface finish achieved in the workpiece was measured.

For drilling, cutting parameters were defined with an experiment $2^{3}$, as shown in Table 3 . This operation was set with the coolant applied through the tool.

As mentioned before, a DOE $3^{3}$ was used for face milling, and this generates 27 possible combinations of cutting parameters for the machining operation. All the values of the low, medium, and high levels for face milling of Table 3 are within the ranges suggested by the tool supplier. A DOE $2^{3}$ was selected for drilling, which implies 9 possible combinations of cutting parameters. Again, all the values of the low, medium, and high levels for face milling of Table 3 are within the ranges suggested by the tool supplier. The results were used for the power demand and energy consumption statistical models proposed by the authors as described below.

\section{Results}

4.1. Tare Power for Face Milling. The statistical software Minitab ${ }^{\circledR}$ was used to carry out the nonlinear and linear regressions of the data obtained with the experiments shown in Table 3. Each one of the 27 defined tests were carried out once, to calculate the constants for equations (10) and (11). Table 4 shows the data of the nonlinear analysis solved with the Levenberg-Marquardt method and based on the results of the facing experiments using the $\mathrm{CNC}$ machine operating with a dynamic toolpath strategy. Table 4 also includes the estimated parameter values and their standard error.

The nonlinear predictive model equation (10), which is based on equation (9), presents $\mathrm{kW}_{\text {Tare }}$ as a function of $n$, spindle speed, and $v_{f}$, feed rate. In the following equation, $\mathrm{kW}_{\text {Tare }}$ is the tare power (i.e., constant power plus variable power):
TABLE 4: Nonlinear regression analysis carried out with the Levenberg-Marquardt algorithm.

\begin{tabular}{lcc}
\hline Parameter & Estimate & SE estimate \\
\hline$x_{1}$ & 0.003014 & 0.003773 \\
$x_{2}$ & 0.639114 & 0.123164 \\
$x_{3}$ & 0.070207 & 0.078683 \\
\hline
\end{tabular}

$p$ value: 0.623 ; maximum iterations: 200 ; tolerance: 0.00001 ; SE: standard error.

$$
\mathrm{kW}_{\text {Tare }}=0.00301386 \cdot n^{0.63914} \cdot v_{f}^{0.0702071} .
$$

Figure 4 shows over the $X$-axis the three spindle speeds chosen in Table 2. However, each speed is repeated three times because it was necessary to make nine tests in this experiment. For example, the spindle speed of $2005 \mathrm{rpm}$ and the feed rate of $802 \mathrm{~mm} / \mathrm{min}$ were tested with the three toolpaths chosen: dynamic, zigzag, and one-way; this is what the first three bars show in Figure 4, and the next three bars show the same spindle speed but with a feed rate of $2647 \mathrm{~mm} / \mathrm{min}$ and the same toolpath mentioned. The tare power over the $y$ axis, of the CNC machine, increases as the spindle speed goes up. The three spindle speeds and feed rate values specified by Table 2 were tested in the experiment. The values calculated with equation (10) to predict the tare power demand for the strategy dynamic are represented by the dotted line.

\subsection{Case 1 Face Milling: Power Demand, Energy} Consumption, and Cycle Time for Toolpath Evaluation. Three different toolpath strategies, i.e., cutting options, were tested with the face milling operation using the medium values defined in Table 3, the cutting parameters were tested three times, and Table 5 shows the average values obtained.

As shown in Table 5, the toolpath dynamic consumed $0.121 \mathrm{kWh}$, which was the lowest value, and the quality surface obtained was $0.22 \mu \mathrm{m}$. Therefore, considering the power and energy values, the dynamic cutting option resulted in being the most convenient for energy saving.

4.3. Nonlinear and Linear Equations Evaluation Using DOE. Table 6 presents the results of the statistical analysis for the experiment defined in Table 3. Twenty-seven tests were 


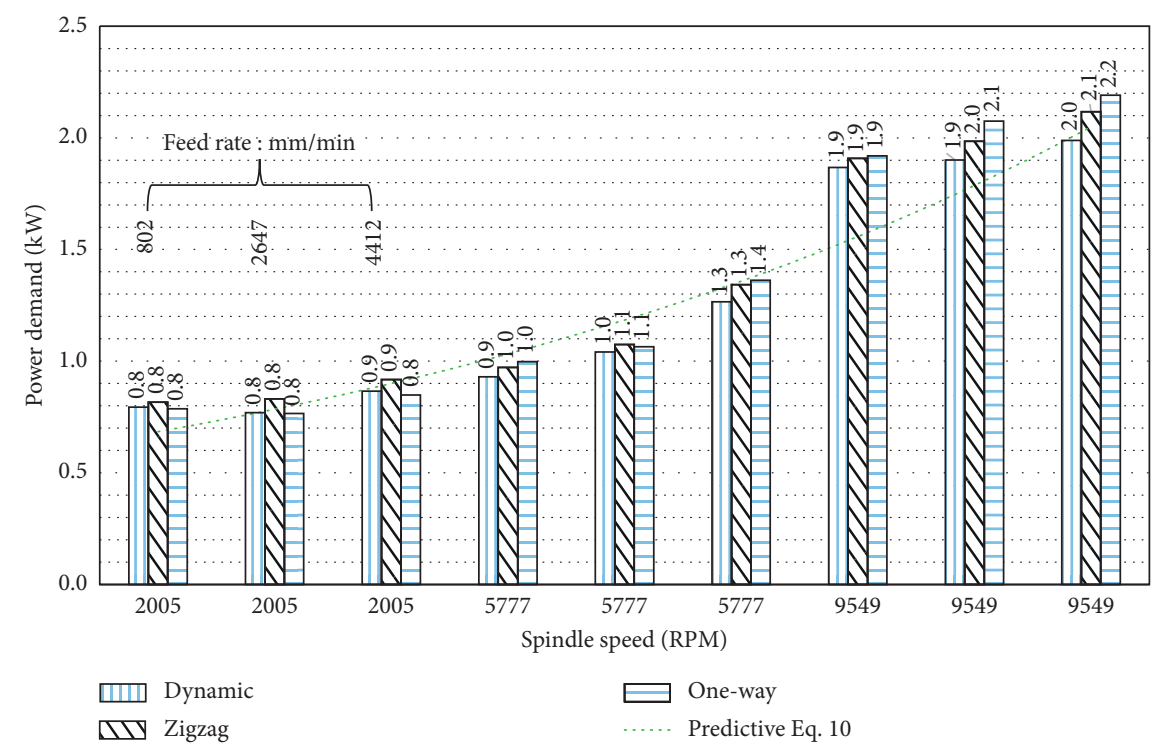

FiguRE 4: Experimental and theoretical tare power for the face milling operation.

TABLE 5: Face milling operation comparative with different cutting options.

\begin{tabular}{lcccc}
\hline Cutting option & Cycle time (hrs) & Power demand $(\mathrm{kW})$ & Energy consumption $(\mathrm{kWh})$ & \multicolumn{2}{c}{ Quality surface } \\
& & & & $R_{a}(\mu \mathrm{m})$ \\
$Y$-axis
\end{tabular}

TABLE 6: Nonlinear regression analysis carried out with the Levenberg-Marquardt algorithm.

\begin{tabular}{lcc}
\hline Parameter & Estimate & SE estimate \\
\hline$x_{1}$ & 0.119379 & 0.0296690 \\
$x_{2}$ & 0.586379 & 0.0127844 \\
$x_{3}$ & 0.660086 & 0.0414910 \\
$x_{4}$ & 0.501722 & 0.0290065 \\
\hline
\end{tabular}

Maximum iterations: 200; tolerance: 0.00001; SE: standard error.

carried out using the dynamic cutting options because it had the lowest power demand. The maximum power demand measured in the experiments was $4.10 \mathrm{~kW}$, the medium value was $2.98 \mathrm{~kW}$, and the lowest was $1.98 \mathrm{~kW}$.

As mentioned in the previous section, the results of Table 6 were used for the model presented by equation (11), where $\mathrm{kW}_{\text {Total }}$, the total power demand, can be estimated from the cutting parameters $a_{p}, v_{c}$, and $f_{z}$ :

$$
\mathrm{kW}_{\text {Total-facing }}=0.119379 \cdot a_{p}^{0.586379} \cdot v_{c}^{0.660086} \cdot f_{z}^{0.501722} \text {. }
$$

Figure 5 shows that there is a normal distribution because the residual model falls on the straight line, which can be seen in the normal probability plot. Additionally, the histogram shows that the data are skewed, and the residuals are randomly distributed, which means constant variance is possible as seen in the versus fits chart. The residuals are independent from one another and are randomly distributed around the center line which means a normal behavior in the versus order chart. This tendency demonstrates the suitability of the experimental results.

Table 7 summarizes the results of the 27 experiments carried out with the DOE specified in Table 3 and shows the ANOVA data. The significance of each factor $a_{p}, v_{c}$, and $f_{z}$ was tested. The analysis was carried out with a confidence level of $95 \%$ for all intervals. In Table 7 , the $p$ values of the three factors are significant because they are lower than 0.05 , which means that the three factors are determinant for the power demand.

The surface quality produced by the 27 tests carried out, which combined different cutting parameters, was measured. The minimum average surface roughness obtained, $R_{a}$, was $0.01 \mu \mathrm{m}$ and the maximum $0.183 \mu \mathrm{m}$. Therefore, these values according to indication of surface texture in technical product documentation fell between $\mathrm{N1}$ and $\mathrm{N} 3$, which represent superfinishing and high level of finish [33].

Equation (12) shows the linear regression equation to predict the power in $\mathrm{kW}$. This includes the coefficients determined using the least squares estimation, which represents the estimated change in mean response for each unit change in the predictor value:

$$
\mathrm{kW}_{\text {Total-facing }}=-2.296+1.1881 a_{p}+0.005681 v_{c}+8.621 f_{z} \text {. }
$$




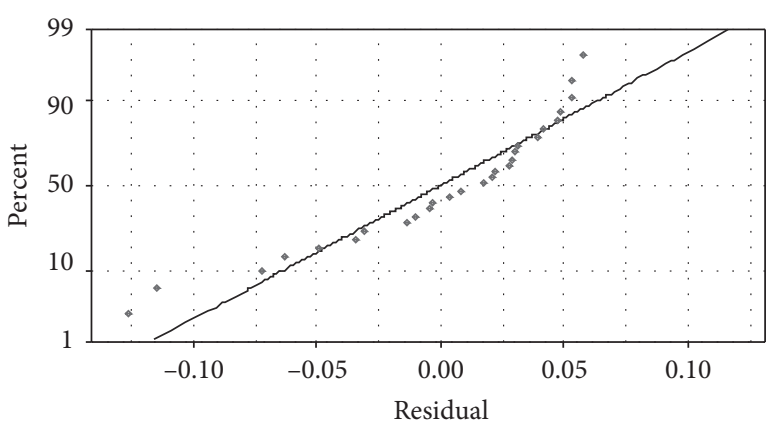

(a)

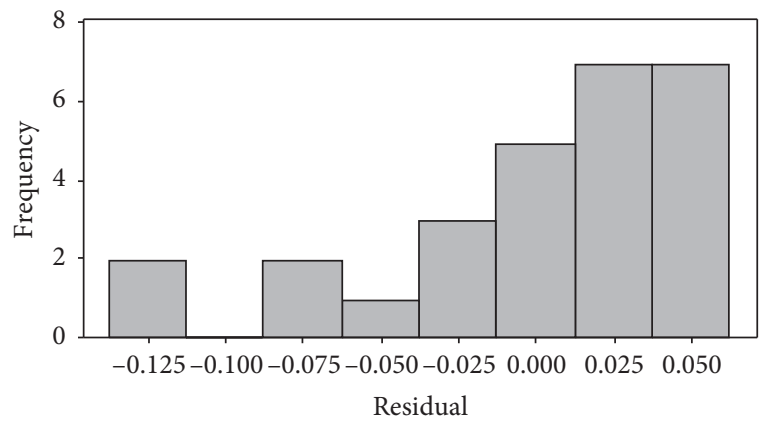

(c)

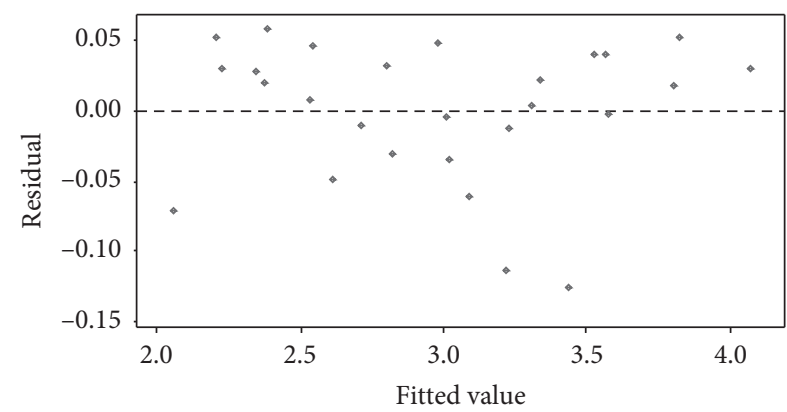

(b)

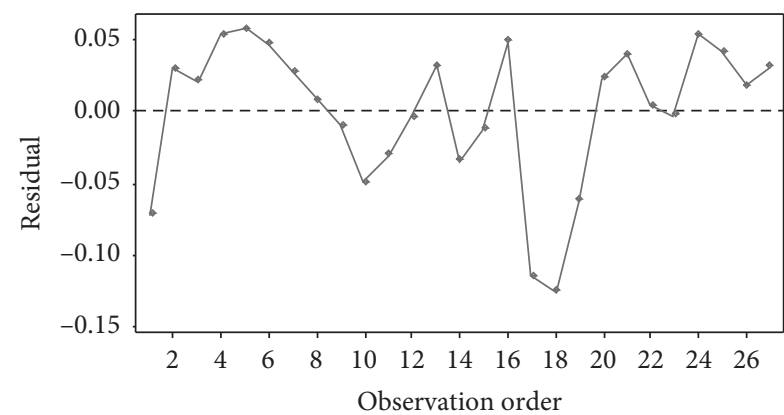

(d)

FIGURE 5: Residual plots for the face milling operation: (a) normal probability plot; (b) versus fits; (c) histogram; (d) versus order.

TABLE 7: Variance analysis of the experiment (Table 3).

\begin{tabular}{lccccc}
\hline Source & DF & Adj. SS & Adj. MS & $F$ value & $p$ value \\
\hline Regression & 3 & 7.89958 & 2.63319 & 669.94 & 0.00001 \\
$a_{p}$ & 1 & 6.35191 & 6.35191 & 1616.07 & 0.00001 \\
$v_{c}$ & 1 & 0.71156 & 0.71156 & 181.04 & 0.00001 \\
$f_{z}$ & 1 & 0.83611 & 0.83611 & 212.72 & 0.00001 \\
Error & 23 & 0.09040 & 0.00393 & & \\
Total & 26 & 7.98998 & & & \\
\hline
\end{tabular}

$R^{2}$ (pred): $98.36 \%$.

Figure 6 shows a comparison between the real power demands (denoted with green color in figure), with the linear equation prediction (equation (12), denoted with blue line in figure) and with the nonlinear equation prediction (equation (11), denoted with yellow color in figure). The figure compares the values obtained in each one of the 27 tests carried out during the experiment. Clearly, the green line is completely aligned with the yellow line, whereas the blue line shows different slight values in comparison with the real power behavior. For example, in test 2, the real value is $2.25 \mathrm{~kW}$, which contrasts with the $1.97 \mathrm{~kW}$ estimated by the linear predictive equation.

4.4. Case 2 Drilling: Power Demand, Energy Consumption, and Cycle Time for Toolpath Evaluation. The two different drilling cutting options tested, counterbore and chip break, were compared. The tool machined the hole with a single movement when the counterbore cutting option was used. The hole was machined progressively, i.e., with a series of penetration and withdrawal movements, using the chip break option.

The toolpath counterbore consumed $0.011 \mathrm{kWh}$ to produce a hole with a radius of $7.001 \mathrm{~mm}$ (Table 8). Therefore, considering the lower energy value, the counterbore toolpath option resulted to be just more convenient for energy saving.

4.5. Nonlinear and Linear Equations Evaluation Using DOE for Counterbore. Table 9 presents the results of the statistical analysis for the experiment defined in Table 3. Nine tests were carried out using the counterbore drilling option because it had the lowest power demand. The maximum power demand measured in the experiments was $4.12 \mathrm{~kW}$, the medium value was $3.46 \mathrm{~kW}$, and the lowest was $2.94 \mathrm{~kW}$.

The modelling of the drilling operation was carried out for the drill counterbore toolpath based on the results of the regression analysis presented in Table 9 . In the model of the operation, $\mathrm{kW}_{\text {Total }}$ represents the total power demand and it is a function of the cutting drilling parameters $v_{c}$ and $f_{n}$, as follows:

$$
\mathrm{kW}_{\text {Total-drilling }}=0.27747 \cdot v_{c}^{0.486936} \cdot f_{n}^{0.0197716} \text {. }
$$

Figure 7 shows that there is a normal distribution because the residuals for the model fall on the straight line, making it possible to see this in a normal probability plot. Also, the histogram shows that the data are skewed, and the residuals are randomly distributed which means constant variance is possible seeing in versus fits charts; the residuals are independent from one another, those randomly around the center line mean a normal behavior in versus order chart. 


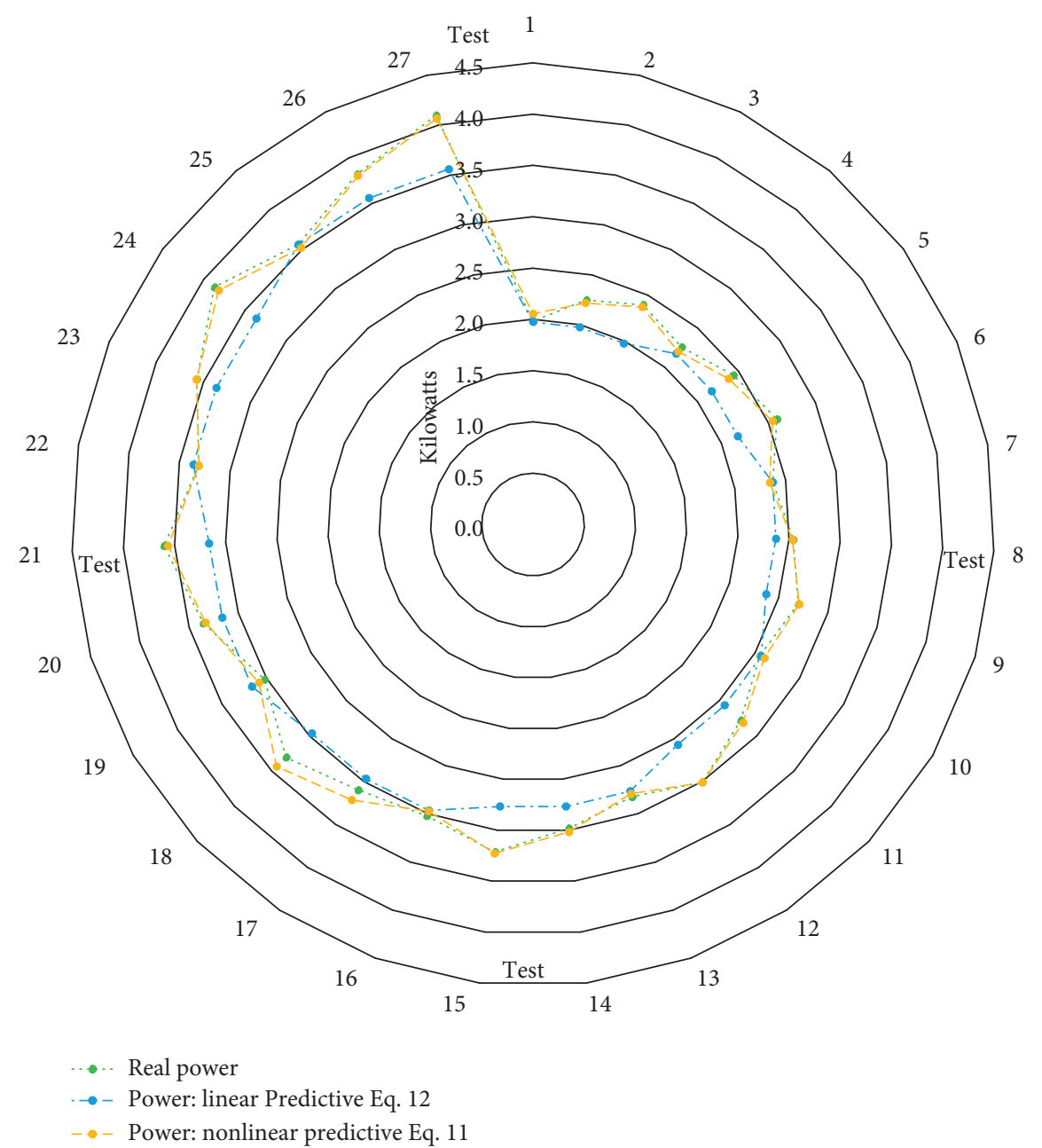

Figure 6: Face milling power real value compared against the results obtained with the nonlinear and linear predictive models.

TABLE 8: Drilling operation comparative with different cutting options.

\begin{tabular}{lcccc}
\hline Cutting option & Cycle time $(\mathrm{hrs})$ & Power demand $(\mathrm{kW})$ & Energy consumption $(\mathrm{kWh})$ & Radii $(\mathrm{mm})$ \\
\hline Counterbore & 0.003 & 3.930 & 0.011 & 7.001 \\
Chip break & 0.003 & 3.256 & 0.014 & 7.002 \\
\hline
\end{tabular}

TABLE 9: Nonlinear regression analysis carried out with the Levenberg-Marquardt algorithm.

\begin{tabular}{lcc}
\hline Parameter & Estimate & SE estimate \\
\hline$x_{1}$ & 0.277470 & 0.0820493 \\
$x_{2}$ & 0.486936 & 0.0552161 \\
$x_{3}$ & 0.019772 & 0.0419983
\end{tabular}

Maximum iterations: 200; tolerance: 0.00001 .

This tendency demonstrates the suitability of the experimental results. The data collected are the support to construct the model of equation (13) with its respective variables.

Table 10 shows the results of the 9 tests carried out with the parameters of the DOE displayed in Table 3 and also shows the ANOVA data. The significance of each factor $v_{c}$ and $f_{n}$ was tested. The analysis was carried out with a confidence level of $95 \%$ for all intervals. The $p$ value of the $v_{c}$ factor is significant, which means that this parameter has an important impact on power. However, the $f_{n}$ factor is higher than 0.05 , implying a low impact on power.

Equation (14) shows the model to predict the power generated with the regression analysis. It can be seen that drilling operations include two cutting parameters, cutting speed $\left(v_{c}\right)$, and feed per revolution $\left(f_{n}\right)$ :

$$
\mathrm{kW}_{\text {Total-drilling }}=1.661+0.008873 v_{c}+0.367 f_{n} \text {. }
$$

Figure 8 shows a comparative between the measured values of power, real powers (green color), with the values obtained with the nonlinear equation (equation (13), denoted with blue color in figure) and with the linear equation (equation (14), denoted with yellow color in figure). The blue line is aligned with the yellow line which are the predictive equations, whereas green line shows slightly 


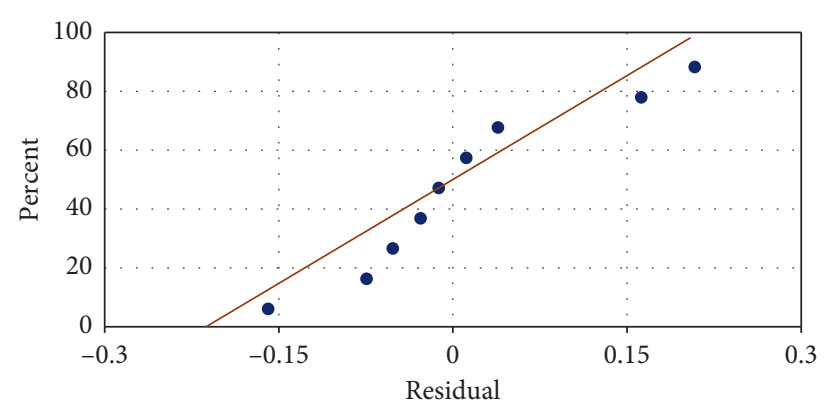

(a)

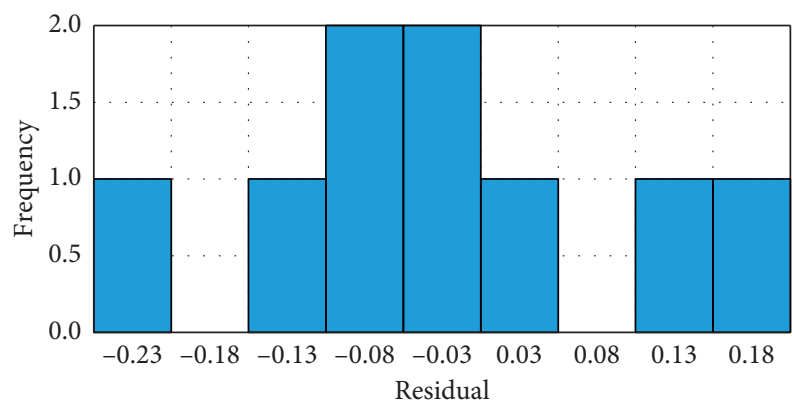

(c)

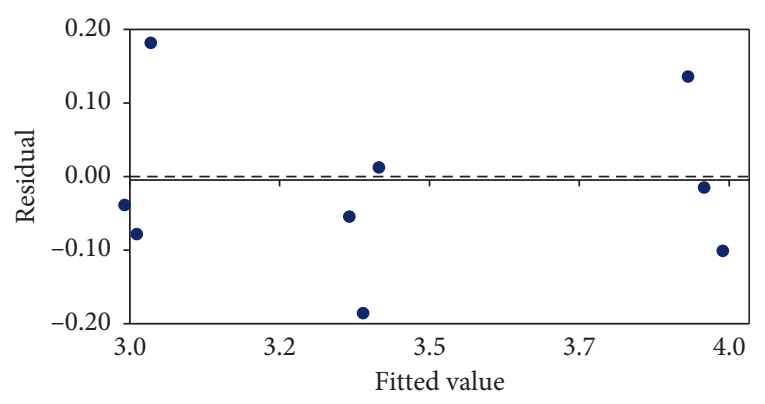

(b)

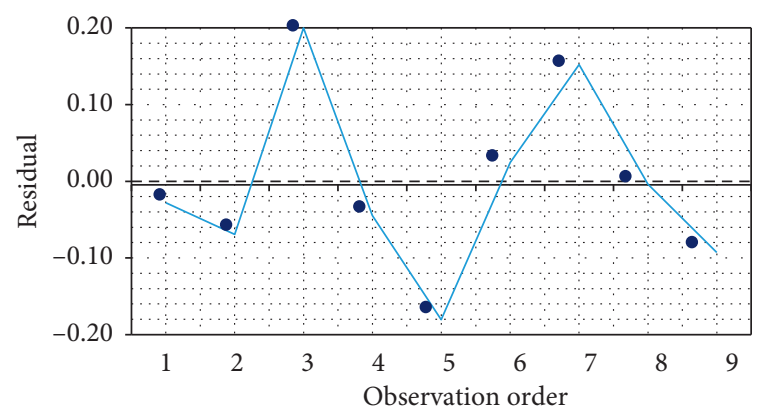

(d)

FIgURE 7: Residual plots of the drilling operation: (a) normal probability plot; (b) versus fits; (c) histogram; (d) versus order.

TABLe 10: Variance analysis.

\begin{tabular}{lccccc}
\hline Source & DF & Adj. SS & Adj. MS & $F$ value & $p$ value \\
\hline Regression & 2 & 1.47609 & 0.73805 & 47.09 & 0.00001 \\
$v_{c}$ & 1 & 1.46422 & 1.46422 & 93.42 & 0.00001 \\
$f_{n}$ & 1 & 0.01187 & 0.01187 & 0.76 & 0.418 \\
Error & 6 & 0.09404 & 0.01567 & & \\
Total & 8 & 1.57013 & & & \\
\hline
\end{tabular}

$R^{2}$ (pred): $93.45 \%$.

different values in tests 3 and 7 . For example, the real value in test 3 is $3.23 \mathrm{~kW}$, in contrast to $3.03 \mathrm{~kW}$ obtained with the linear predictive equation and $3.07 \mathrm{~kW}$ with the nonlinear equation.

\section{Discussion}

Tool path strategies and cutting parameters are important factors in the power and energy behavior. It is also known that the maximum power demanded by a CNC machine is tare power [7], but it is not constant. However, if the tool path does not change, the spindle speed and the feed rate will vary depending on cutting parameters. This could be modelled with linear and nonlinear equations, in the idle CNC machine tool condition. The work reported in this paper considered the dynamic tool path strategy for facing as a basis for developing a model because its power demand was inferior to the other two milling strategies (Table 5). There was no representative difference of the surface finish obtained with each strategy, as displayed in Table 4; along the $X$-axis, $R_{a}$ varied up to $0.02 \mu \mathrm{m}$ and up to $0.04 \mu \mathrm{m}$, in the $Y$ direction.
As the tool path was constant in facing, the energy performance depended on the spindle speed and feed rate. Tare power was modelled with the dynamic tool path. However, the same procedure reported in this paper can be used with other machining processes and different tool paths to define the appropriate model for tare power. A nonlinear equation was used to represent the tare power as a function of cutting parameters because of the comparison of the results obtained with linear and nonlinear models reported in Figures 5 and 7.

The data collected through different experiments allowed the authors to choose cutting parameters to achieve workpiece design specifications and, at the same time, take into account low energy consumption operations. According to the data collected using different tool paths for facing, the dynamic tool path reported $1.52 \mathrm{~kW}$ and $0.121 \mathrm{kWh}$, the zigzag $1.55 \mathrm{~kW}$ and $0.154 \mathrm{kWh}$, and the one-way tool path reported $1.99 \mathrm{~kW}$ and $0.136 \mathrm{kWh}$. This behavior was determinant to select the results of the dynamic tool path strategy to define the model for the operation because of its low power demand.

From the face milling 27 experiments, the cutting parameters of the three tests $(A, B$, and $C)$ carried out with the dynamic toolpath with the lowest power demand and energy consumption were as follows: $A-a_{p}=1 \mathrm{~mm}, v_{f}=350 \mathrm{~m} /$ min, and $f_{z}=0.20 \mathrm{~mm}$ per tooth; $B-a_{p}=1.5 \mathrm{~mm}$, $v_{f}=350 \mathrm{~m} / \mathrm{min}$, and $f_{z}=0.20 \mathrm{~mm}$ per tooth; and $\mathrm{C}-a_{p}=2 \mathrm{~mm}, v_{f}=350 \mathrm{~m} / \mathrm{min}$, and $f_{z}=0.20 \mathrm{~mm}$ per tooth. The power, energy, and roughness surface were $A=2.59 \mathrm{~kW}$, $0.030 \mathrm{kWh}$, and $0.085 \mu \mathrm{m} ; B=3.21 \mathrm{~kW}, 0.038 \mathrm{kWh}$, and $0.56 \mu \mathrm{m}$; and $C=3.87 \mathrm{~kW}, 0.046 \mathrm{kWh}$, and $0.66 \mu \mathrm{m}$. Based on these results, the values for $v_{f}$ and $f_{z}$ could be selected, 


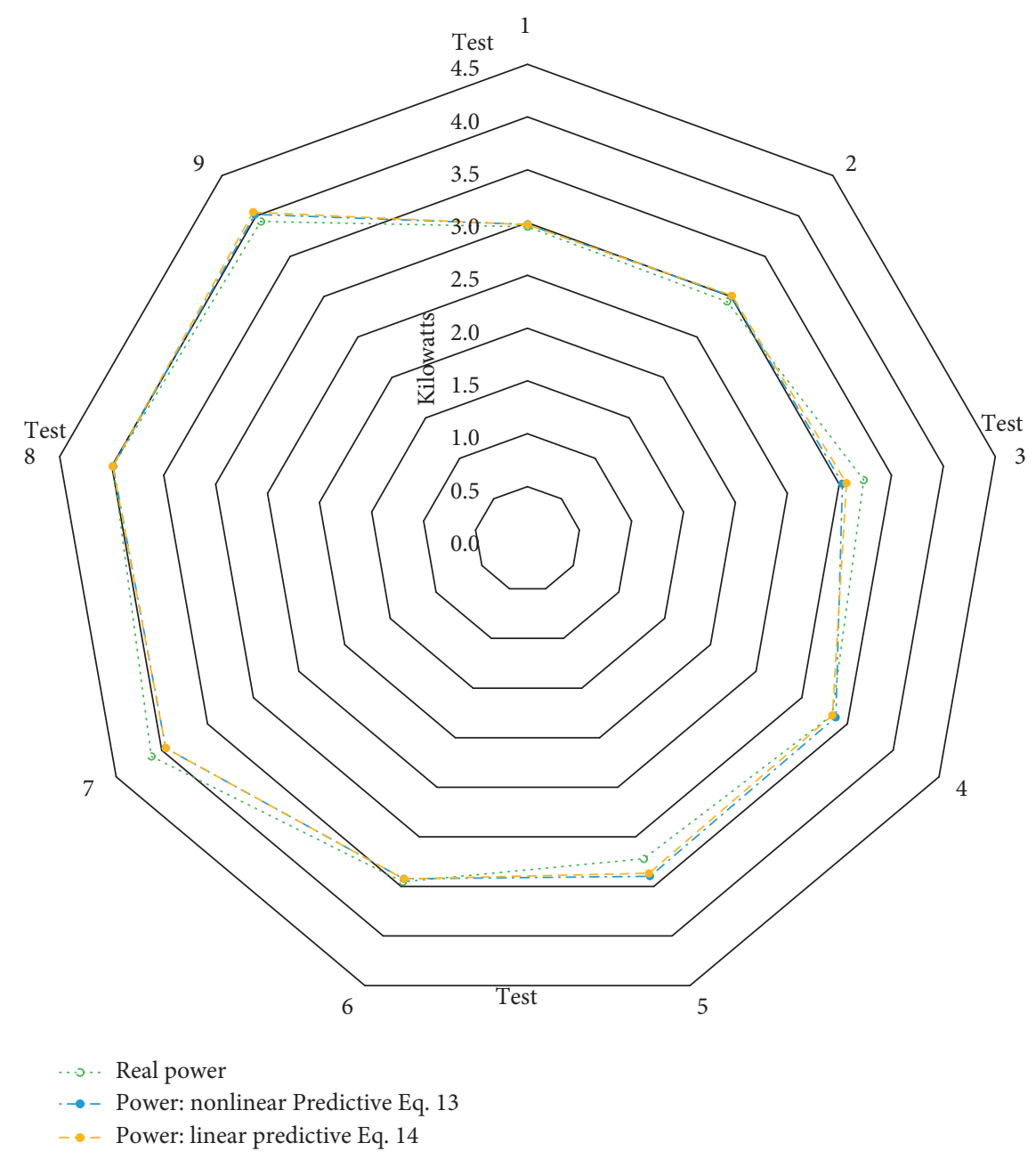

FIGURE 8: Drilling power real value compared against the results obtained with the nonlinear and linear predictive models.

and there are two options for $a_{p}$ : the lowest power and energy consumption; therefore, the lowest cycle time was obtained with option $A$, but the best quality surface was produced with option $C$. So, if the surface finish achieved with $A$ satisfies the workpiece requirements, this is the best option. Still, $C$ is also adequate.

Drilling was tested with coolant applied to the tool, especially for removing the chip. In addition, the highest variation in ratio was $0.08 \mathrm{~mm}$. However, drill counterbore required the highest value in power with $2.9 \mathrm{~kW}$, reaching $3.9 \mathrm{~kW}$, and on the contrary, this operation showed the lowest energy value with $0.009 \mathrm{kWh}$ until reaching $0.011 \mathrm{kWh}$. Besides, the same behavior observed in the drill counterbore operations was found using the chip break strategy. Power values ranged from $2.8 \mathrm{~kW}$ to $3.2 \mathrm{~kW}$ and energy from $0.01 \mathrm{kWh}$ to $0.014 \mathrm{kWh}$. The results abovementioned showed that, in the case of drill counterbore, the variation between the lowest and the highest power was estimated in $0.97 \mathrm{~kW}$ and the figure for energy was estimated in $0.002 \mathrm{kWh}$. When drilling with chip break, the power was $0.376 \mathrm{~kW}$ and the energy $0.004 \mathrm{kWh}$. The power variation was an important measurement in this operation, especially because this behavior is a consequence of all power peaks that take place during machining.

\section{Conclusions}

Based on the comprehensive outcome of our investigation, the following conclusions can be drawn:

(1) The approach used in this research in terms of cutting parameters as demonstrated by the nonlinear power model is validated by the fact that the $p$ value for the lack-of-fit test is 0.623 (Table 4), which means that this value is larger than the significance level of 0.05 or the linear model with $R^{2}$ (pred) 98.36 (Table 7).

(2) The power demand and energy consumption differences of the facing and drilling operations for the workpiece analyzed varied from 2 to $4 \mathrm{~kW}$ and $0.125 \mathrm{kWh}$ to $0.011 \mathrm{kWh}$. In order to achieve a good balance of consumption, i.e., without big differences or peaks, it is necessary to carefully select the toolpath trajectory, cutting parameters, and cutting tools 
for each machining operation required by a workpiece.

(3) Different behaviors in energy consumption derived from choosing one or another toolpath available in commercial software. The values found for dynamic toolpaths (tare power) are $1.21 \mathrm{kWh}$, as a minimum, to $1.54 \mathrm{kWh}$, as a maximum. This could represent an optimization value of $0.21 \%$ (Table 5). Moreover, regarding the behaviors in energy consumption shown when using one or another cutting parameters, the values found for dynamic toolpaths are $0.030 \mathrm{kWh}$, as a minimum, to $0.046 \mathrm{kWh}$, as a maximum (Discussion). This could mean an optimization value of $0.35 \%$.

Further research will focus to find a correct energy balance, using equation prediction by varying the cutting parameters. More studies are necessary in order to better fit the quality of surface with energy consumption.

\section{Abbreviations}

$\begin{array}{ll}a_{p}: & \text { Depth of cut } \\ \text { DOEs: } & \text { Design of experiments } \\ f_{n}: & \text { Feed per revolution } \\ f_{z}: & \text { Feed per tooth } \\ \mathrm{kW}: & \text { Kilowatts } \\ \mathrm{kWh}: & \text { Kilowatts hour } \\ n: & \text { Spindle speed } \\ R_{\mathrm{a}}: & \text { Roughness average } \\ \mathrm{rpm}: & \text { Revolutions per minute } \\ v_{c}: & \text { Cutting speed } \\ v_{f}: & \text { Feed rate } \\ \text { ANOVA } R^{2} \text { (pred): } & \text { Analysis of variance } R^{2}, \text { predicted. }\end{array}$

\section{Data Availability}

The nature of the data obtained in the present investigation are of the experimental type. The equations presented in the article are of the own authorship of all those who participated in the writing of the same and come from the result of studying the theories related to the design of experiments. Addition information can be made available from the correspondence author (xavier_snk@hotmail.com) or the principal author (gminquiz@yahoo.com) upon request. There is no restriction to access such data.

\section{Conflicts of Interest}

The authors declare that they have no conflicts of interest.

\section{Acknowledgments}

The authors thank the support provided by Sandvik Coromant S.A. de C.V. (México) to carry out the experiments; in particular, the collaboration of Pablo Flores is appreciated. The research reported in this paper was sponsored by the Universidad Nacional Autónoma de México (Grant nos. UNAM-DGAPA-PAPIIT IT101816 and 101718), Consejo Nacional de Ciencia y Tecnología (Grant
288411 and support: 355272), and Project SEP-CONACyTBasic Science (no. 83239).

\section{References}

[1] U. S. Administration, "Energy information," Monthly Energy Review Journal, vol. 35, no. 5, 2017.

[2] T. N. Nghiep, A. Sarhan, and A. A. D. Hideki, "Analysis of tool deflection errors in precision CNC end milling of aerospace aluminum 6061-T6 alloy," Measurement, vol. 125, pp. 476495, 2018.

[3] L. Aoyama, Strengthened Manufacturing Research at NAMRC 45, SME, Dearborn, MI, USA, 2017, https://www.sme.org/ technologies/articles/2017/may/strengthened-manufacturingresearch-at-namrc-45/.

[4] T. Gutowski, J. Dahmus, and A. Thiriez, "Electrical energy requirements for manufacturing processes," in Proceedings of the 13th CIRP International Conference of Life Cycle Engineering, Leuven, Belguim, May 2006.

[5] Y. Guo, J. Loenders, J. Duflou, and B. Lauwers, "Optimization of energy consumption and surface quality in finish turning," Procedia CIRP, vol. 1, pp. 512-517, 2012.

[6] P. T. Mativenga and M. F. Rajemi, "Calculation of optimum cutting parameters based on minimum energy footprint," CIRP Annals, vol. 60, no. 1, pp. 149-152, 2011.

[7] N. Diaz, S. Choi, M. Helu et al., Machine Tool Design and Operation Strategies for Green Manufacturing, Laboratory for Manufacturing and Sustainability UC Berkeley, Berkeley, California, 2010.

[8] A. Rangarajan and D. Dornfeld, "Efficient tool paths and Part Orientation for face milling," CIRP Annals, vol. 53, no. 1, pp. 73-76, 2004.

[9] N. Diaz, M. Helu, and D. Dornfeld, "Design and operation strategies for green machine tool development," Laboratory for Manufacturing and Sustainability, Berkeley, CA, USA, 2010.

[10] K. Erkorkmaz, S. E. Layegh, I. Lazoglu, and H. Erdim, "Feedrate optimization for freeform milling considering constraints from the feed drive system and process mechanics," CIRP Annals, vol. 62, no. 1, pp. 395-398, 2013.

[11] A. Aramcharoen and P. T. Mativenga, "Critical factors in energy demand modelling for CNC milling and impact of toolpath strategy," Journal of Cleaner Production, vol. 78, pp. 63-74, 2014.

[12] G. M. Minquiz, V. Borja, M. Lopez-Parra, A. C. RamírezReivich, M. A. Domínguez, and A. Alcaide, "A comparative study of CNC part programming addressing energy consumption and productivity," in Proceedings of the 6th CIRP International Conference on High Performance Cutting, pp. 581-586, Springer, Berlin, June 2014.

[13] R. Lmalghan, M. C. K. Rao, S. ArunKumar, S. S. Rao, and M. A. Herbert, "Machining parameters optimization of AA6061 using response surface methodology and Particle Swarm optimization," International Journal of Precision Engineering and Manufacturing, vol. 19, no. 5, pp. 695-704, 2018.

[14] X. Luan, S. Zhang, and G. Li, "Modified power prediction model based on infinitesimal cutting force during face milling process," International Journal of Precision Engineering and Manufacturing-Green Technology, vol. 5, no. 1, pp. 71-80, 2018.

[15] D. Y. Pimenov, "Mathematical modeling of power spent in face milling taking into consideration tool wear," Journal of Friction and Wear, vol. 36, no. 1, pp. 45-48, 2015. 
[16] D. Y. Pimenov, A. Bustillo, and T. Mikolajczyk, “Artificial intelligence for automatic prediction of required surface roughness by monitoring wear on face mill teeth," Journal of Intelligent Manufacturing, vol. 29, no. 5, pp. 1045-1061, 2018.

[17] N. Kumar Sahu and A. B. Andhare, "Modelling and multiobjective optimization for productivity improvement in high speed milling of Ti-6Al-4V using RSM and GA," Journal of the Brazilian Society of Mechanical Sciences and Engineering, vol. 39, no. 39, pp. 5069-5085, 2017.

[18] L. Peña-Parás, D. Maldonado Cortés, M. Rodriguez-Villalobos, A. G. Romero Cantu, and O. E. Montemayor, "Enhancing tool life, and reducing power consumption and surface roughness in milling processes by nanolubricants and laser surface texturing," Journal of Cleaner Production, vol. 253, p. 2020.

[19] T. Behrendt, A. Zein, and S. Min, "Development of an energy consumption monitoring procedure for machine tools," CIRP Annals, vol. 61, no. 1, pp. 43-46, 2012.

[20] M. Helu, J. Rühl, D. Dornfeld, P. Werner, and G. Lanza, "Evaluating trade-offs between sustainability, performance, and cost of green machining technologies," in Proceedings of the 18th CIRP International Conference on Life Cycle Engineering, Braunschweig, Germany, May 2011.

[21] L. Li, C. Li, Y. Tang, and Q. Yi, "Influence factors and operational strategies for energy efficiency improvement of CNC machining," Journal of Cleaner Production, vol. 161, pp. 220-238, 2017.

[22] L. Wen, Efficiency of Manufacturing Processes, Springer, Berlin, Germany, 2015.

[23] S. Kalpakjian and S. Schmid, Manufactura, Ingeniería $Y$ Tecnología, Pearson Education, London, UK, 2006.

[24] G. Quintana, J. Ciurana, and J. Ribatallada, "Modelling power consumption in ball-end milling operations," Materials and Manufacturing Processes, vol. 26, pp. 746-756, 2013.

[25] Z. H. Wang, J. T. Yuan, T. T. Liu, J. Huang, and L. Qiao, "Study on surface roughness in high-speed milling of AlMn1Cu using factorial design and partial least square regression," The International Journal of Advanced Manufacturing Technology, vol. 76, no. 9-12, pp. 1783-1792, 2014.

[26] X. D. Fang and H. Safi-Jahanshahi, "A new algorithm for developing a reference-based model for predicting surface roughness in finish machining of steels," International Journal of Production Research, vol. 35, no. 1, pp. 179-199, 1997.

[27] H. Ganesan and G. Mohankumar, "Optimization of machining techniques in CNC turning centre using genetic algorithm," Arabian Journal for Science and Engineering, vol. 38, no. 6, pp. 1529-1538, 2013.

[28] F. Draganescu, M. Gheorghe, and C. V. Doicin, "Models of machine tool efficiency and specific consumed energy," Journal of Materials Processing Technology, vol. 141, no. 1, pp. 9-15, 2003.

[29] J. Taneja, M. Bector, and R. R. Kumar, "Application of Taguchi method for optimizing turning process by the effects of machining parameters," International Journal of Engineering and Advanced Technology, vol. 2, 2012.

[30] W. M. Ponnala and K. L. N. Murthy, "Modeling and optimization of end milling machining process," International Journal of Research in Engineering and Technology, vol. 1, 2012.

[31] D. C. Montgomery and G. C. Runge, Applied Statistics and Probability for Engineers, United States of America, John Wiley \& Sons, Hoboken, NJ, USA, 2003.

[32] Sandvik, Rotating Tools Milling, Sandvik, Stockholm, Swede, 2012.
[33] ISO 1302:2002, Geometrical Product Specifications (GPS)Indication of Surface Texture in Technical Product Documentation, ISO 1302:2002, Los Angeles, CA, USA, 2012. 